\title{
Findings of Anisometropic Amblyopia in Children
}

\author{
Eglantina Molosiu' ${ }^{1}$, Sulejman Zhugli ${ }^{2}$ \\ ${ }^{1}$ Regional Hospital of Fier, Albania \\ ${ }^{2}$ University Hospital Center "Mother Theresa”, Tirana, Albania;
}

\begin{abstract}
Anisometropic amblyopia is the second most common cause of amblyopia. Anisometropic amblyopia occurs when unequal focus between the two eyes causes chronic blur on one retina. The aim of this study was to describe the treatment of Anisometropic amblyopia in a regional hospital. 192 patients with anisometropic amblyopia presenting for examination at Ofthalmologic Clinic of Regional Hospital of Fier in Albania between 2012 and 2014, were enrolled in the study. The mean age of the patients at study entry was 6.6 \pm 0.9 years (range $3-9$ years); $46 \%$ were female and $75 \%$ were white. Table 1 provides the baseline characteristics of the cohort. The mean visual acuity measurement in the amblyopic eyes at study entry was $3 / 10$ in the right eye and in the left eye was 4/10. At the initial 5-week visit, visual acuity improvement from the spectacle-corrected baseline acuity averaged $1.9 \pm 1.4$ lines, with $54 \%$ of the patients having improvement of 2 or more lines of acuity and 10\% meeting the definition for resolution. If spectacles are not tolerated other option should be considered as, contact lenses, refractive surgery of cornea, etc.
\end{abstract}

Keywords: Anisometropic amblyopia, treatment, children, examination

\section{Introduction}

Anisometropic amblyopia is the second most common cause of amblyopia. Anisometropic amblyopia occurs when unequal focus between the two eyes causes chronic blur on one retina. Anisometropic amblyopia can occur with relatively small amounts of asymmetric hyperopia or astigmatism. Generally, larger amounts of anisomyopia are necessary for amblyopia to develop. Anisometropic amblyopia is an insidious disease because unlike strabismic amblyopia, the eyes appear normal to an observer. Anisometropic amblyopia is most commonly caught by vision screening either in a pediatrician's office or in the school system. The prevalence of amblyopia is reported as $2-4 \%$ in North America and is the primary cause of unilateral vision loss in children (1). According to Hess et al., one third of amblyopia cases are caused by anisometropia, one third by strabismus and one third from a combination of the two (2). However, other authors have reported that $50 \%$ of cases with amblyopia are caused by anisometropia (3). As previously discussed, anisometropic amblyopia can occur when there is a difference in refractive error between the two eyes. It is generally accepted that pathological changes from amblyopia occur primarily in the primary visual cortex and lateral geniculate nucleus. Experiments by Hubel and Wiesel were the first to demonstrate neuronal loss in the primary cortex (V1) using experimental deprivation amblyopia in kittens (4). Subsequent amblyopia studies in animals have shown neurological changes in layer IVc of V1 and the lateral geniculate nucleus (5). Recent studies have verified that there is significant loss of neural activity in the visual cortex. However, the ocular dominance columns do not appear to shrink in size in patients with strabismic or anisometropic amblyopia (6). Treatment of anisometropic amblyopia starts with eliminating the competitive advantage of the dominant eye. This is usually done by prescribing the cyclopegic refraction to the child for full time wear. Sometimes children will not tolerate their full hyperopic correction and so symmetric decreases in plus lens power may be required. Some children will respond to refractive correction alone. For those with residual amblyopia after refractive correction, the next step is optical penalizations for the dominant eye. There are a number of techniques for optical penalization including occlusion (both part time and full time), placement of filters, refractive defocus, and pharmacologic blurring. The prognosis for treatment varies significantly based on the age of the child and the type of treatment initiated (7). In general, treatment is more successful if the child is treated at a younger age. Although refractive amblyopia is more commonly associated with anisometropic hyperopia, unilateral high myopia tends to have a worse prognosis. The aim of this study was to describe the treatment of Anisometropic amblyopia in a regional hospital.

\section{Material and Methods}

192 patients with anisometropic amblyopia presenting for examination at Ofthalmologic Clinic of Regional Hospital of Fier in Albania between 2012 and 2014,were enrolled in the study. Screening Visit and Prescription of Spectacles We enrolled patients age 3 to $<7$ years with a history of untreated anisometropic amblyopia For this study of anisometropic amblyopia, patients were excluded if they had any measurable heterotropia in primary gaze at distance or near fixation in their prescribed spectacles or a documented history of strabismus. Testing for eccentric fixation to detect a microtropia with identity was not required. Spectacles were prescribed based on a cycloplegic refraction using cyclopentolate $1 \%$. Anisometropia, astigmatism, and myopia were fully corrected. Hyperopia gt;3.00 D spherical equivalent was either fully corrected or symmetrically under-corrected by no more than $+1.50 \mathrm{D}$ in both eyes. Hyperopia $\leq 3.00 \mathrm{D}$ spherical equivalent was corrected at investigator discretion.

\section{Baseline Examination}

The prescribed spectacles were not worn prior to the day of the baseline examination, which occurred within 30 days of the screening visit. After the spectacles had been worn for 10-30 minutes, corrected visual acuity was measured in each eye by a study-certified vision tester using the electronic visual acuity tester. This test provides visual acuity scores in one 1 line increments. To be included in the study, it was 


\section{International Journal of Science and Research (IJSR) \\ ISSN (Online): 2319-7064 \\ Index Copernicus Value (2013): 6.14 | Impact Factor (2014): 5.611}

necessary for the spectacle-corrected amblyopic eye visual acuity to be between 20/40 and 20/400 inclusive, the sound eye acuity to be $20 / 40$ or better, and the interocular acuity difference to be 3 or more lines. If the baseline visual acuity was worse than the screening visual acuity in a patient with hyperopic refractive error, acuity testing was repeated with a $-1.00 \mathrm{D}$ lens to determine if an inability to relax accommodation sufficiently to accept the prescribed hyperopic spectacles was responsible for the worsening of vision. If testing with the $-1.00 \mathrm{D}$ lens improved acuity, a symmetrically reduced hyperopic correction was prescribed and the patient did not wear any spectacles until 10-30 minutes prior to the rescheduled baseline examination visit. Follow-up Visits

Protocol-specified follow-up visits were conducted every 5 $( \pm 1)$ weeks as long as the amblyopic eye acuity (1) had improved at least one line from the prior visit and (2) was still at least one line worse than the sound eye acuity. At every visit, visual acuity was measured in each eye and if amblyopic eye visual acuity had not improved at least one line from the prior 5-week visit, the amblyopic eye was retested. Follow up was complete when neither measurement of the amblyopic eye acuity showed improvement of at least one line from the prior visit. Amblyopia was considered "resolved" in patients achieving an interocular acuity difference of 1 line or less; these patients continued using their spectacle correction, did not have other treatment prescribed, and had a final follow up visit 4-6 months later. At the time of stabilization, patients who had an interocular difference of 2 or more lines entered the randomized trial phase described in a companion report. Data from the patients randomized to the control group are included in this report to assess the proportion of patients who showed additional visual acuity improvement with continued spectacle wear after having not shown improvement over the prior 5-week period. For each patient, the maximum acuity improvement was computed and the visit at which this occurred was identified. For purposes of analysis, resolution of amblyopia was defined as improvement of the amblyopic eye acuity to be no more than one line worse than the sound eye. The proportion of patients whose amblyopia resolved was computed and the $95 \%$ confidence interval calculated.

\section{Results and Discussion}

The mean age of the patients at study entry was $6.6 \pm 0.9$ years (range $3-9$ years); $46 \%$ were female and $75 \%$ were white. Table 1 provides the baseline characteristics of the cohort. The mean visual acuity measurement in the amblyopic eyes at study entry was 3/10 in the right eye and in the left eye was $4 / 10$. At the initial 5 -week visit, visual acuity improvement from the spectacle-corrected baseline acuity averaged $1.9 \pm 1.4$ lines, with $54 \%$ of the patients having improvement of 2 or more lines of acuity and $10 \%$ meeting the definition for resolution, amblyopic eye acuity no more than one line worse than sound eye. Visual acuity improvement continued beyond the initial 5 weeks of spectacle wear for 88 (46\%) of the 192 patients completing the initial 5-week visit, with the longest duration of improvement being 27 weeks in one patient. The change from the spectacle-corrected baseline acuity to the best acuity achieved at any visit averaged $2.4 \pm 1.7$ lines, with
$128(67 \%)$ of the patients having improvement of 2 or more lines of acuity and $41(21 \%) 95 \$,$% CI 15.4 \%$ to $27.4 \%$ ) having resolution of their amblyopia. Among the 41 patients meeting the resolution criteria, the amblyopic eye was one line worse than the sound eye in $23(56 \%)$ and the same or better than the sound eye in $18(44 \%)$.

Anisometropic amblyopia has been reported to occur with as little as one diopter difference between the two eyes (8). In recent years, therapy with glasses alone has become more acceptable and has been shown to improve vision $\geq 2$ lines in $77-93 \%$ of patients with complete resolution of amblyopia in $27-45 \%$ (9). Treatment effect appears to plateau around 2 months. If severe amblyopia is present at presentation (defined by the Amblyopia Treatment Study Group as visual acuity 20/100 20/400), patching or pharmacological penalization may be added. However, patching or atropine must be considered in addition to optical management if patients show no improvement with glasses alone after 3-5 months. It has been thought that visual maturity occurs between the ages of 6 and 9 years. However, a growing number of studies are showing a response to amblyopia treatment in older patients (10). In 2005, was reported that amblyopia improved with optical correction alone in about one fourth of patients aged 7 to 17 years, with $15 \%$ of patients improving when first treated in their teens (11). This supports the need for treatment through the first decade of life and possibly into the second decade of life.

Patient compliance with glasses wear is a frequent challenge in the treatment of anisometropic amblyopia. It is especially difficult in very young patients as they are often unable to notice a subjective improvement in their vision with the glasses on. Discussion with the parents about the need for continued glasses wear and the risk of recurrence is important to successful treatment as can be demonstrated by the slip in visual acuity in our patient. It is important for parents to understand that continued spectacle correction is critical until visual maturity even when apparent stability has been achieved.There are few life-altering diseases in medicine with a better cost/benefit ratio in terms of treatment. The challenge is to be able find the affected children. Effective screening and early diagnosis are key public health concerns in addressing anisometropic amblyopia as late diagnosis and poor initial acuity portend a poor response to treatment (12). Use of the Bruckner test is an easy and effective method for detecting anisometropia by pediatricians and other non-ophthalmic professionals. This can be done quickly in the office and prompts a referral to an ophthalmologist or optometrist. Large-scale screening efforts have been successful at identifying amblyopia risk factors for a large percentage of children (13). This screener has a high positive predictive value for anisometropia (96\%). Amblyopia continues to be a significant public health problem, affecting $2 \%$ to $5 \%$ of the population. When diagnosed and treated early in life, the visual loss may be reversed, which supports the need for early identification and treatment of anisometropia. Visual acuity improvement with refractive correction alone in children with anisometropic amblyopia has been observed in both retrospective and pilot studies $(14,15)$. However, only recently has the magnitude and time course of this phenomenon been evaluated prospectively reporting a mean

\section{Volume 4 Issue 11, November 2015}




\section{International Journal of Science and Research (IJSR) \\ ISSN (Online): 2319-7064 \\ Index Copernicus Value (2013): 6.14 | Impact Factor (2014): 5.611}

improvement of nearly 3 lines in 18 patients with anisometropic amblyopia treated with spectacles and improvement occurred for an average of 15.6 weeks in their subgroup of patients with anisometropic amblyopia (16). They have termed this effect "refractive adaptation," but we prefer to refer to it as optical treatment of amblyopia. The time course in our study for improvement to best amblyopic eye visual acuity was variable. The majority $(83 \%)$ of patients stopped improving before 15 weeks, but one patient improved for 30 weeks. The finding of visual acuity improvement over many weeks for children with anisometropic amblyopia treated with refractive correction alone can guide (1) clinicians on the expected duration of treatment when using spectacles alone as the treatment and (2) investigators who want to control for the treatment effect of refractive correction when evaluating the effectiveness of other amblyopia treatments. In earlier randomized trials of patching and pharmacological treatment optimum spectacle correction was required for a minimum of 4 weeks prior to enrollment.13-16 The results of the present study suggest that in these previous trials, some of the visual acuity improvement was due to optical treatment of amblyopia with spectacles. Although this would have the effect of reducing the statistical power to detect a treatment group difference, these trials were highly powered and this effect of spectacles therefore should not alter the primary conclusions of those studies.

\section{Conclusion}

Amblyopia continues to be a significant public health problem, affecting $2 \%$ to $5 \%$ of the population. When diagnosed and treated early in life, the visual loss may be reversed, which supports the need for early identification and treatment of anisometropia. If spectacles are not tolerated other option should be considered as, contact lenses, refractive surgery of cornea,etc.

\section{References}

[1] Levi, D.M, McKee, S.P, Movshon, J.A. Visual deficits in Anisometropia. Vision Research 2011;51:48-57.

[2] Steel AL, Bradfield YS, Kushner BJ et al. Successful treatment of anisometropic amblyopia with spectacles alone. J AAPOS 2006;10(1):37-43.

[3] Pediatric Eye Disease Investigator Group: Treatment of anisometropic amblyopia in children with refractive correction. Ophthalmology 2006;113:895-903.
[4] Pediatric Eye Disease Investigator Group. Randomized trial of treatment of amblyopia in children aged 7 to 17 years. Arch Ophthalmol. 2005;123(4):437-447.

[5] Pediatric Eye Disease Investigator Group. Patching vs atropine to treat amblyopia in children aged 7 to 12 years: a randomized trial. Arch Ophthalmol 2008; 12:1634-42.

[6] Pediatric Eye Disease Investigator Group. A randomized trial comparing Bangerter filters and patching for the treatment of moderate amblyopia in children. Ophthalmology 2010;17(5):998-1004.

[7] Leguire LE, Komaromy KL, Nairus TM, Rogers GL. Long-term follow-up L-dopa treatment in children with amblyopia. J Pediatr Ophthalmol Strabismus 2002;39:326-330.

[8] Pediatric Eye Disease Investigator Group. The clinical profile of moderate amblyopia in children younger than 7 years. Arch. Ophthalmol 2002; 120:281-287.

[9] Chen P, et al. Anisometropic amblyopia treated with spectacle correction alone: possible factors predicting success and time to start patching. Am. J. Ophthalmol 2007; 143: 54-60.

[10] Menon,V. Shailesh G, Sharma P, Saxena R. Clinical trial of patching versus atropine penalization for the treatment of anisometropic amblyopia in older children. J AAPOS 2008; 12:493-497.

[11] Scheiman MM, et al. Randomized trial of treatment of amblyopia in children aged 7 to 17 years. Arch. Ophthalmol 2005; 123:437-447.

[12] Hussein MAW, Coats DK, Muthialu A, Cohe, E, Paysse EA. Risk factors for treatment failure of anisometropic amblyopia. J AAPOS 2004; 8: 429-434.

[13] Longmuir SQ, et al. Nine-year results of a volunteer lay network photoscreening program of 147809 children using a photoscreener in Iowa. Ophthalmology 2010; 117: $1869-1875$

[14] Astle WF, Rahmat J, Ingram AD, Huang PT. Laserassisted subepithelial keratectomy for anisometropic amblyopia in children: outcomes at 1 year. J Cataract Refract Surg 2007; 33: 2028-2034.

[15] Birch E, Holmes J. The clinical profile of amblyopia in children younger than 3 years of age. J AAPOS 2010; 14: 494-497.

[16] Stewart CE, Moseley MJ, Fielder AR, et al. Refractive adaptation in amblyopia: quantification of effect and implications for practice. $\mathrm{Br} \mathrm{J}$ Ophthalmol 2004;88:1552-6. 


\section{International Journal of Science and Research (IJSR)}

ISSN (Online): 2319-7064

Index Copernicus Value (2013): 6.14 | Impact Factor (2014): 5.611

Table 1: Baseline characteristics of the cohort of children

\begin{tabular}{|c|c|c|}
\hline Variables & $\mathbf{N}$ & $\%$ \\
\hline Age M (SD) & $6.6(0.9)$ & \\
\hline \multicolumn{3}{|l|}{ Family history } \\
\hline Yes & 7 & 3.6 \\
\hline No & 185 & 96.4 \\
\hline \multicolumn{3}{|l|}{ Titmus test } \\
\hline Gross stereopsis & 13 & 6.8 \\
\hline Abnormal & 21 & 10.9 \\
\hline Absent & 6 & 3.1 \\
\hline Normal & 152 & 79.2 \\
\hline Not established & 2 & 1.0 \\
\hline \multicolumn{3}{|l|}{ Worth test } \\
\hline Abnormal & 44 & 22.9 \\
\hline Normal & 148 & 77.1 \\
\hline \multicolumn{3}{|l|}{ Foria } \\
\hline Yes & 1 & 0.5 \\
\hline No & 188 & 97.9 \\
\hline \multicolumn{3}{|l|}{ Strabismus } \\
\hline Yes & 43 & 22.4 \\
\hline No & 149 & 77.6 \\
\hline \multicolumn{3}{|l|}{ Ocular movement } \\
\hline Abnormal & 5 & 2.6 \\
\hline Normal & 187 & 97.4 \\
\hline \multicolumn{3}{|l|}{ Nystagmus } \\
\hline Yes & 2 & 1.0 \\
\hline No & 190 & 99.0 \\
\hline \multicolumn{3}{|c|}{ External examination } \\
\hline Abnormal & 5 & 2.6 \\
\hline Normal & 187 & 97.4 \\
\hline \multicolumn{3}{|l|}{ Bio microscopy } \\
\hline Abnormal & 4 & 2.1 \\
\hline Normal & 188 & 97.9 \\
\hline \multicolumn{3}{|l|}{ Fundus oculi } \\
\hline Abnormal & 2 & 1.0 \\
\hline Normal & 190 & 99.0 \\
\hline
\end{tabular}

Volume 4 Issue 11, November 2015 